\title{
Cyanobacterial diversity through culture-dependent and independent approaches in Chapada das Mesas National Park, Maranhão, Brazil
}

\section{Ana Carolina de Araújo Butarelli}

Federal University of Maranhao: Universidade Federal do Maranhao

Lucas Salomão de Sousa Ferreira

Federal University of Maranhao: Universidade Federal do Maranhao

\section{Raquel Riyuzo}

University of Sao Paulo Institute of Chemistry: Universidade de Sao Paulo Instituto de Quimica

Hivana Melo Barbosa Dall'Agnol

Federal University of Maranhao: Universidade Federal do Maranhao

\section{Carlos Morais Piroupo}

University of Sao Paulo Institute of Chemistry: Universidade de Sao Paulo Instituto de Quimica

\section{Aline Maria da Silva}

University of Sao Paulo Institute of Chemistry: Universidade de Sao Paulo Instituto de Quimica

\section{João Carlos Setubal}

University of Sao Paulo Institute of Chemistry: Universidade de Sao Paulo Instituto de Quimica

Leonardo Teixeira DallAgnol ( $\sim$ leonardo.td@ufma.br)

Federal University of Maranhao: Universidade Federal do Maranhao https://orcid.org/0000-0003-08378836

\section{Research Article}

Keywords: Culture Enrichment, Hidden Diversity, Photosynthetic Microbiome, Sediments

Posted Date: January 31st, 2022

DOI: https://doi.org/10.21203/rs.3.rs-1254910/v1

License: (c) (i) This work is licensed under a Creative Commons Attribution 4.0 International License. Read Full License 


\section{Abstract}

The Chapada das Mesas National Park (CMNP) is a Conservation Unit in brazilian Cerrado biome, which is considered a hotspot for biodiversity conservation and possess important rivers, waterfalls and springs with economical and touristic importance. This study objective was to characterize the total and microbial photosynthetic community of the CMNP from surface sediment samples from waterfalls of two touristic sites: Pedra Caída and Farinha river. These samples were cultivated in selective medium for photosynthesizers and both the raw samples and the enriched ones were submitted to DNA extraction and sequencing of the V3-V4 hypervariable region of the $16 \mathrm{~S}$ rRNA gene on the lon Torrent platform. The reads were analyzed using QIIME2 software and the Phyloseq package. The enrichment allowed detecting and identifying many genera of cyanobacteria in the Chapada das Mesas National Park, which would probably not be possible without the combination of approaches. A total of 34 genera of photosynthetic microorganisms were classified in the samples from the consortia, highlighting "Chloroplast" (45.60\%) and Synechocystis_CCALA_700 (10.10\%) as the most abundant groups in the tropical alkaline lakes of Maranhão. As for the functional analysis, metabolic functions associated with methanotrophy and methylotrophy, hydrocarbon degradation, phototrophy and nitrogen fixation were predicted. The results highlight a great diversity of photosynthetic microorganisms in Cerrado and the importance of using a combination of approaches when analyzing target groups who usually are underrepresented like photosynthetizers.

\section{Introduction}

The Chapada das Mesas National Park (CMNP) is a Conservation Unit located south of Maranhão in the northeast region of Brazil, covering a total area of 15995162 hectares (Brasil 2012). The CMNP covers a significant area of the Cerrado, a biome considered as hotspot and threatened by desertification in the east, which has already lost about $80 \%$ of its natural cover. In addition, the park is home to the main rivers in the region, dozens of waterfalls, and more than 400 springs which attracts tourists and has socio-environmental relevance. This richness in water resources and biodiversity with tropical climate, makes CMNP a very favorable environment photosynthetic microorganisms, especially cyanobacteria.

The Chapada das Mesas National Park is located in an area that acts as an ecotone between three important biomes (Cerrado, Amazon, and Caatinga), making it a unique and extremely important area for biodiversity conservation (Ibama 2013). Owing to its humid tropical climate with high temperatures throughout the year, the park is home to a wide range of microorganisms related to ecosystem processes that may be linked to climate change (Cavicchioli et al. 2019). Tropical environments favor cyanobacterial growth, as high temperatures of water bodies promote the proliferation of cyanobacterial blooms (Richardson et al. 2018), a characteristic that can be mainly driven by the anthropogenic eutrophication of fresh waters (Taranu et al. 2015). Microbial communities present in sediments are responsible for most of the metabolic activity in river ecosystems (Gibbons et al. 2014). Understanding the structure of microbial communities in various water environments associated with waterfalls can help us predict how these ecosystems will change in response to human practices in these regions, as the CMNP has a strong tourist appeal.

Cyanobacteria plays a fundamental role in the ecological balance of freshwater bodies and contributes to stabilize the substrate in the biogeochemical flow of oxygen, carbon, and nitrogen, in addition to being the 
primary producers in the food chain (Rigonato et al. 2012). Despite being one of the most varied groups of photosynthetic prokaryotes in terms of morphology, physiology, and metabolism, there is still a lack of studies on their diversity (Dvorák et al. 2015; Azizan et al. 2020).

The taxonomy of cyanobacteria has been historically challenging. The traditional way of evaluating their communities involves analyzing morphological characters using microscopy (Bhadury and Singh 2020). However, this method proved to be insufficient to discern lines with more subtle differences, in addition to other problems, such as the fact that the culture media we have available reproduce only a small cultivable fraction of microbial diversity (Stevenson et al. 2004). Studies involving the cultivation of photosynthetic microorganisms are also limited due to obstacles in the stages of purification and isolation, making it difficult to obtain axenic cultures (Ferris and Hirsch 1991). Cyanobacteria have even more particularities, are often associated with heterotrophic organisms and organic matter in the environment, and may present changes in their metabolism and morphology under cultural stress conditions (Zhubanova et al. 2013).

Advances in DNA sequencing techniques have provided access to prokaryotic genetic information available in environmental samples (Tan et al. 2015) and the approach that encompasses the use of marker gene sequences, such as the 16S rRNA gene, allows a considerable part of cultivable (Appolinario et al. 2016) and non-cultivable (Hugenholtz et al. 2016) microorganisms to be identified. However, it is possible to face obstacles such as the limited detection of microorganisms in low abundance in the environment, the difficulty in distinguishing specific taxonomic levels (Rego et al. 2019) or even the fraction of sequences not yet registered (Rinke et al. 2013).

An alternative to circumvent these restrictions is the blending of dependent and independent cultivation techniques to enable the identification of microbial diversity and its interest groups (Fournier et al. 2015; Pudasaini et al. 2017; Zehavi et al. 2018; Sarhan et al. 2019). However, there is considerable divergence between the sets of bacteria identified by each approach, indicating not only the limitations existing in each of them, but also the possibility of combining their strengths to obtain more complete information.

Cyanobacteria, despite belonging to a phylum that includes organisms that are important for their metabolic capacity, it is still a difficult group to study, especially in microbiome studies. This characteristic is associated with slow growth, difficulty in obtaining axenic cultures, and challenges in the study of mixed cultures through next-generation sequencing. In this context, this work aims to characterize the structure of microbial communities representing the Chapada das Mesas National Park, with emphasis on the phylum Cyanobacteria, and demonstrate the importance of combining dependent and independent cultivation techniques in studies involving groups such as photosynthetic microorganisms.

\section{Materials And Methods}

\subsection{Sampling sites}

Sediment samples from the bottom of the water body (approximately $10 \mathrm{~cm}$ above) were collected from 10 th to 11 th March 2017, in Chapada das Mesas National Park (CMNP) and adjacent areas during the rainy season. Six points were sampled in waterfalls of the Pedra Caída Tourist Complex (resort) (E1-E6), located on 
the outer edge of the CMNP, and six in the Prata and São Romão waterfalls along the Farinha River (E7-E12), totaling 12 sampling points (Supplementary Table 1 and Supplementary Figure 1). These points were chosen because they are places of great tourist influx, with varying degrees of difficulty in accessing and very different physicochemical characteristics. Geographical coordinates were obtained using a differential GPS device AG-132 (OmniSTAR).

The sediment was collected using a sterile spatula and stored in a $50 \mathrm{~mL}$ conical falcon tube. The material was preserved on ice and refrigerated $\left(4^{\circ} \mathrm{C}\right)$ for transport until further inoculation of the material in BG-11 culture medium. The time between collection and inoculation did not exceed $72 \mathrm{~h}$. Sediment aliquots were separated and frozen at $-20^{\circ} \mathrm{C}$ for further environmental DNA extraction.

Water samples were collected in $1500 \mathrm{~mL}$ sterile plastic bottles and kept on ice during transport for further quantification of nitrates, nitrites, total solids, and phosphorus.

\subsection{Physicochemical analysis}

The physicochemical analysis of water just above the sediment was performed using a multiparameter probe (Horiba W-20XD) to measure, in situ, temperature, $\mathrm{pH}$, dissolved oxygen (DO), and turbidity of the water body adjacent to the collected sediment. Samples were collected in $1500 \mathrm{~mL}$ sterile plastic bottles and kept on ice during transport for the quantification of nitrates, nitrites, total solids, and phosphorus.

\subsection{Culture dependent approach: enriched microbiome}

The culture medium used for the growth of photosynthetic microorganisms was BG-11. The culture medium was autoclaved for $20 \mathrm{~min}$ at $121^{\circ} \mathrm{C}$. The cultivation of photosynthetic microorganisms was carried out following the protocol described by Ferris and Hirsch (1991). Each sediment sample collected was inoculated in the culture medium, receiving the nomenclature of $\mathrm{C} 1-\mathrm{C} 6$ referring to samples E1-E6 collected in Pedra Caída and C7-C12 referring to samples E7-E12 collected from Farinha River. The enrichment was carried out in $250 \mathrm{~mL}$ Erlenmeyer flasks containing $100 \mathrm{~mL}$ of sterile BG-11 culture medium at a ratio of 1:10 (w:v), and incubated in an incubator with photoperiod, with a schedule of $13 \mathrm{~h}$ light and $11 \mathrm{~h}$ dark, at a temperature of $28^{\circ} \mathrm{C}$. Three inoculations were carried out, with an interval of 1 month between each one, to stabilize the microbial consortium in the flasks and to obtain sufficient biomass for subsequent DNA extraction. Cultivation was not intended to obtain axenic cultures, which is notoriously difficult for this group of microorganisms, as our aim was to enrich the culture with photosynthetic microorganisms, especially from the group of cyanobacteria and chlorophyte microalgae.

\subsection{DNA extraction and microbiome sequencing}

Metagenomic DNA extractions were performed from two sets of samples: (i) in natural sediment samples (called the environmental microbiome) and (ii) non-axenic cultures enriched in photosynthesizers, obtained after 3 months of replenishing (called photosynthetic consortia microbiome). Extraction was performed using the PowerSoil DNA Isolation Kit (MO BIO Laboratories Inc., Carlsbad, CA, USA) following the manufacturer's instructions. The purity of the extracted DNA was evaluated using a NanoDrop 2000/2000c spectrophotometer (Thermo Fisher). 
The variable regions V3 and V4 of the 16S rRNA gene were amplified using primers 515F-m (5'GTGYCAGCMGCCGCGGTAA-3') and 806R-m (5'-GGACTACNVGGGTWTCTAAT-3') (Wang and Qian 2009). The $\mathrm{PCR}$ reaction was performed with a final volume of $24 \mu \mathrm{L}$ containing: $13.3 \mu \mathrm{L}$ of deionized water, $0.5 \mathrm{mM}$ of dNTPs, $2.5 \mathrm{mM}$ of $\mathrm{MgCl}_{2}, 0.2 \mathrm{mM}$ of each primer, $1 \mathrm{U}$ of Taq DNA Polymerase, and $2.510 \times$ buffer and approximately $50 \mathrm{ng}$ of DNA from each sample. The reaction was carried out in a Veriti ${ }^{\circledR}$ 96- Well Thermal Cycler (Thermo Fisher) for $5 \mathrm{~min}$ at $95^{\circ} \mathrm{C}$, followed by $30 \mathrm{~s}$ at $95^{\circ} \mathrm{C}, 30 \mathrm{~s}$ at $55^{\circ} \mathrm{C}$, and $120 \mathrm{~s}$ at $72^{\circ} \mathrm{C}$ for 35 cycles, followed by $10 \mathrm{~min}$ at $72^{\circ} \mathrm{C}$.

The amplicon library was prepared and sequenced according to the standards and protocols of the Brazilian Microbiome Project (BMP - http://www.brmicrobiome.org/) on the lon Torrent platform (Thermo Fisher Scientific, Massachusetts, USA) in the Federal University of Pampa (UNIPAMPA).

Sequencing data has been deposited at the National Center for Biotechnology Information Sequence Read Archives under BioProject ID PRJNA783649 (See Supplementary Table 14).

\subsection{Bioinformatic analysis}

The raw reads obtained through sequencing were analyzed for their quality using the FASTQC software (Andrews 2010), and were kept for the subsequent analysis of sequences with Phred Score $\geq 25$, on average, a criterion that was also used to determine the cut-off size of the sequences at $290 \mathrm{bp}$.

The pipeline used for bioinformatics analysis was made available by the Brazilian Microbiome Project (BMP). Raw data were analyzed using the Quantitative Insights into Microbial Ecology (QIIME) software pipeline, version 2 (QIIME2, release 2021.1) (Bolyen et al. 2019). The sequences were initially imported (qiime tools import), summarized (qiime demux summarize), and analyzed for their quantity and feasibility. Denoising was performed using the DADA2 plugin (qiime dada2 denoise-pyro) (Callahan et al. 2016). The lon Torrent data were submitted to the command "qiime dada2 denoise-pyro single-end" (parameters: -p-trunc-len 290 -p-trimleft 15). At this stage, the readings were subjected to error correction, filtration, trimming, chimeras' removal, and grouping into amplicon sequence variants (ASV, 100\% similarity limit). The taxonomic composition of the unique sequences was determined using the pre-trained Naive Bayes classifier (qiime feature-classifier fitclassifier-naive-bayes) against the SILVA version 138 database (Quast et al. 2013). The phylogenetic matrix was generated using the command "qiime phylogeny align-to-tree-mafft-fasttree".

\subsection{Statistical analysis}

Statistical analysis was performed using R software (version 4.0.3) using the Phyloseq package (McMurdie and Holmes 2013). Alpha diversity metrics (observed ASVs, chao1 and Shannon) were calculated based on the relative abundance of ASVs. The Shapiro-Wilk test was used to analyze the normality of the data. Data that had a normal distribution were tested using the $t$-test, and those that were not normally distributed were tested using the Mann-Whitney test. The distributions of diversity indices are plotted in a box-plot. Beta diversity analysis was performed using the Bray-Curtis metric to analyze the dissimilarity between Farinha River and Pedra Caída in both treatments. Then, the difference in the composition of microbial communities, using physicochemical factors (Supplementary Table 4), was tested for significance based on a permutation test (999 interactions) using the envifit function (vegan package). The nonparametric permutation analysis of variance (PERMANOVA) was used to compare the dissimilarity of the microbial composition using the 
ANOSIM package. Non-metric multidimensional scaling (nMDS) was used to visualize the ordering of samples. All diversity metrics were calculated using the R vegan package (Oksanen et al. 2019) and all plots were generated using the ggplot2 package (Wickham 2016). Significantly more abundant ASVs between samples from Farinha River and Pedra Caída between the two treatments were identified using the "DESeq2" package by differential abundance analysis (Love et al. 2014). Statistical significance was set at $p<0.05$.

\subsection{Functional inference}

Predictions of ecological processes based on metataxonomy were performed using the FAPROTAX tool (Louca et al. 2016). The FAPROTAX_1.2.4 database is available at http://www.zoology.ubc.ca/louca/FAPROTAX. We removed from the output FAPROTAX table the categories that were not applied in our study and those where the score was equal to zero.

\section{Results And Discussion}

\subsection{Sequencing effectiveness}

Sequencing on the lon Torrent platform of the 16S rRNA gene generated 5,206,899 reads of good quality from a total of 24 samples, where the representatives of the environmental microbiome (E1-E12) and enriched microbiome (C1-C12) had 1,162,255 and 4,044,644 reads, respectively (Supplementary Table 2 and 3). The sequences were clustered into 4,792 ASVs. The number of ASVs detected per sample ranged from 4,792 to 2,537 (Supplementary Table 4). The rarefaction curves indicated a plateau, suggesting sufficient sequencing depth to analyze the diversity of the microbial community in the samples (Supplementary Figure 2).

\subsection{Abiotic factors}

The water adjacent to the collected sediment was characterized according to environmental factors: (i) turbidity ranged from 3.97 (sample E3) to $22.3 \mathrm{NTU}$ (E10); (ii) variable temperature from $25^{\circ} \mathrm{C}$ (E8) to $28.2^{\circ} \mathrm{C}$ (E4); $\mathrm{pH}$ ranged from 6.9 (points $\mathrm{E} 6, \mathrm{E} 7$ and E8) to 7.4 (E11 and E12); (iv) $\mathrm{NH}_{3}, \mathrm{NO}^{-2}, \mathrm{NO}^{-3}, \mathrm{PO}_{3}^{-4}$ and $\mathrm{SiO}_{2}$ ranged from 6.76 (E6 and E12) to $112.56 \mu \mathrm{mol} \mathrm{L}^{-1}$ (E12), from 0.02 (E1) to $0,24 \mu \mathrm{mol} \mathrm{L}^{-2}$ (E8), 2.20 (E7) to $58.07 \mu \mathrm{mol} \mathrm{L}^{-3}$ (E8), 0.02 (E1 and E5) to $1.21 \mu \mathrm{mol} \mathrm{L}^{-4}$ (E3) and 0.57 (E5) to $4.30 \mu \mathrm{mol} \mathrm{L}^{-5}$ (E12), respectively. A table that includes all the physicochemical data collected is available in Supplementary Table 5.

A comparative analysis (Mann-Whitney test) of the variables between the two sites showed significant differences in turbidity $(p=0.003), \mathrm{NO}^{-2}(p=0.003), \mathrm{SiO}_{2}(p=0.037)$, and temperature $(p=0.003)$ (See Supplementary Table 6). The samples from Pedra Caída showed low turbidity, $\mathrm{NO}^{-2}$, and $\mathrm{SiO}_{2}$, and a higher temperature than the samples from Farinha River. Samples from Pedra Caída (E1-E6), which has greater anthropogenic influence, showed higher values of ammonia, indicating a possible influence of evictions related to tourist activity.

\subsection{Structural diversity of microbial communities}

\subsubsection{Environmental microbiome}


The taxonomic composition of sequences from environmental samples identified 10 phyla $(>1 \%)$. The most abundant representatives of the community were Proteobacteria (55.84\%), Firmicutes (10.95\%), Acidobacteria (9.77\%), Verrucomicrobiota (5.64\%), Actinobacteria (5.78\%), Desulfobacterota (4.86\%), Bacteroidetes (2.0\%), Chloroflexi and Myxococcota (both representing 1.87\%), and Cyanobacteria (1.38\%) (Figure 3C and Supplementary Table 8).

The high abundance of the Proteobacteria phylum (55.84\%) (Figure 3C) corroborates other studies carried out in sediments associated with water bodies (Pascault et al. 2014, Zarraonaindia et al. 2015). Members of the phylum play an essential role in biogeochemical cycles, mainly in the nitrogen cycle (Spain et al. 2009). Members of the phylum Firmicutes (10.95\%) are linked to the nitrogen cycle and actively participate in the replacement of this compound in the environment (Anderson et al. 2018; Jung et al. 2013). Acidobacteria $(9.77 \%)$ are physiologically diverse, and the cultivated members of this phylum are heterotrophic and metabolize a wide variety of complex carbohydrates and nitrates (Kielak et al. 2016). The presence of other phyla such as Actinobacteria, Verrucomicrobiota, Bacteroidetes, Chloroflexi, and Cyanobacteria are important bacterioplankton participants that make up freshwater ecosystems (Crump and Hobbie 2005; Allgaier and Grossart 2006; He et al. 2017).

The Cyanobacteria phylum was identified among the ten most abundant groups in environmental samples; however, at a more specific taxonomic level, such as genus, representatives of the cyanobacteria phylum showed low diversity and abundance in relation to other groups. Through the taxonomic composition, only 17 genera were classified, which reveals the difficulty of identifying representatives of this phylum in analysis that encompass the new generation environmental sequencing, even in a freshwater environment and a photic zone, which, theoretically, is an environment that favors the adaptation of photosynthetic species (Richardson et al. 2018).

Cyanobacteria are one of the most diverse groups in the bacterial domain (Gaysina et al. 2018) and, even with advanced next-generation DNA sequencing techniques, there is no high-level sensitivity to the point of recognizing or approaching the total diversity of low-abundance groups in environmental samples (Tromas et al. 2017). In addition, the databases still have a large sequence gap when it comes to cyanobacteria, and Alvarenga et al. (2017) demonstrated that among all genomic sequences deposited in the $\mathrm{NCBI}$, only $1 \%$ is related to the phylum Cyanobacteria.

In general, between the sampling points (Pedra Caída and Farinha River), the richness of ASVs measured by the Chao1 estimator, the diversity estimated by Shannon, and the number of observed ASVs (Figure 1A) did not demonstrate an effect on the estimated indices (Mann-Whitney, $W=8, p=0.1 ; t$-test, $t=-1.4434, \mathrm{df}=6.3$, $p=0.1$; Mann-Whitney, $W=8, p=0.1$; respectively).

The non-metric multidimensional scale (nMDS) based on dissimilarities calculated using the Bray-Curtis metric showed that there is dissimilarity between the microbial communities found in the regions of Rio flour and Pedra Caída (Figure 1B). Based on the multivariate permutation analysis of variance (PERMANOVA), the variation in the structure of the microbial community is, in part, explained by the sample points analyzed (ANOSIM, $R=0.356, p=0.01$ ). 
The analysis carried out through the envifit revealed that part of the microbial community variation can be explained by environmental factors characteristic of each sampled region. Parameters such as temperature (envifit; $R_{2}=0.65 ; p=0.01$ ), NO ${ }^{-2}$ (envifit; $R_{2}=0.58 ; p=0.02$ ), and NO-3 (envifit; $R_{2}=0.68 ; p=0.02$ ) had a significant effect (Supplementary Table 10) on microbial communities, as variations in environmental temperature and nutrient availability can modulate the community and delimit microbial niches (Samad et al. 2020).

\subsubsection{Enriched microbiome}

Studies that characterized the cyanobacterial consortia aimed to analyze their ability to degrade compounds such as crude oils (Hamouda et al. 2016) and hydrocarbons from petroleum (Ichor et al. 2016), or selection of cyanobacteria that can be used biotechnologically for the treatment of effluents (Arias et al. 2017). Unlike the aforementioned approaches, these consortia aimed to favor the growth of photosynthetic microorganisms, selecting them from culture in culture medium (BG-11) for further taxonomic analysis. Thus, it is possible to enrich and study under sampled photosynthetic organisms in sequencing environmental samples.

The microbial composition of the consortia presented seven phyla $(>1 \%)$ of the bacterial domain, according to the classified sequences. The most abundant representatives were Proteobacteria (45.2\%), Cyanobacteria (26.5\%), Bacteroidetes (18\%), Planctomycetes (3.3\%), Verrucomicrobiota (2.5\%), Acidobacteria (2.2\%), and Armatimonadota (1.8\%) (Supplementary Table 9).

Photosynthetic microbial communities have a strong relationship with heterotrophic organisms because of the different ecological roles played by these groups. The carbon secreted by the phytoplankton community promoted the growth of the associated microbial communities. In natural environments, such as Chapada das Mesas, the relationships between cyanobacteria and the heterotrophic community tend to be balanced, which guarantees local ecological stability (Xu et al. 2018). In artificial communities, such as enriched cultures, the presence of these photosynthetic microorganisms makes it possible for the heterotrophic community to grow in minimal culture medium, as they act as primary producers in the microcosm.

Microbial consortia come from the enrichment of environmental samples with the objective of favoring certain target groups through the use of selective culture media for a determined objective. Mixed populations in bacterial cultures may seem undesirable objectives; however, with this approach, it is possible to analyze functions that are difficult to perform or even impossible for individual strains or species (Brenner et al. 2008). Cyanobacteria often have heterotrophic microbes in symbiotic association with their cells, and it is assumed that these associations exist even among the oldest known life forms, which represent important ecological interactions during the evolutionary history of this group (Alvarenga et al. 2017).

By analyzing the taxonomic level of the genera of photosynthetic microorganisms, it was possible to identify a total of 34 different microbial groups, including organisms of the phylum Cyanobacteria and organisms classified as "Chloroplast," which includes the eukaryotic representatives of microalgae, in the samples of the consortia of both the Complex of Pedra Caída and in the Farinha River region. The most abundant genera were "Chloroplast" (45.60\%), Synechocystis_CCALA_700 (10.10\%), Geminocystis_PCC-6308 (9.38\%), YB-42 (7.38\%), Ancylothrix_8PC (4.86\%), JSC-12 (3.99\%), Cyanobacteria (3.62\%), Phormidium_IAM_M-71 (2.91\%), 
Cyanobacterium_PCC-10605 (2.01\%), Pleurocapsa_PCC-7327 (1.71\%), SU2_symbiont_group (1.12\%), and HAVOmat113 (1.06\%) (Figure 2, Supplementary Table 11 and Supplementary Table 12).

Some groups of photosynthetic microorganisms were identified in the environmental microbiome, but at low abundance (Figure 2). The park's photosynthetic diversity has microbial genera that would not be identified using only the cultivation-independent approach, because these groups may be in low abundance in these water bodies, limitations of the technique itself, among others. When the culture-dependent approach was used, cyanobacterial diversity increased by 65\%. Some genera such as Acaryoclhoris, Aetokthonos, Calotrhrix, Chalicogloea, Cyanobium, Gastranaerophilales, Gleobacter, Microseria, Myxosarcina, Scytonema, and SepB.3 were identified only in the environmental analysis and probably failed to compete under controlled cultivation conditions with other microorganisms that were enriched in the consortia.

As the most abundant of the two treatments, the group classified as "Chloroplast" was taxonomically assigned as representative of the phylum Cyanobacteria. The high abundance of this less specifically classified group may represent a need to update the SILVA 138 database for this group (Lesack and Birol 2018), because many phytoplankton representatives have not yet been identified, and they may also be related to microalgae that were probably enriched in the consortia and are present in the natural environment, as there is a strong association between the presence of these other phytoplankton components and the enrichment of cyanobacteria (Perera et al. 2019).

The identification of the genus Pantanalinema in the consortia is interesting, as this group was first recorded in saline-alkaline lakes and was characterized by its ability to survive and produce biomass at a $\mathrm{pH}$ ranging from 4 to 11 (Vaz et al. 2015). It was mainly described based on the phylogenetic information of its 16S rRNA sequences (Genuário et al. 2018). Microorganisms from this group have already been identified in Amazonian rivers and in the Cerrado Maranhense, are part of a group of bacteria that cannot be cultivated in isolation, and later recovered from an association with cnidarians (Genuário et al. 2018; Ferreira et al. 2021). The first genome of the genus was recently characterized (Ferreira et al. 2021), and the analysis revealed a still unknown environmental and biotechnological potential. Brazil has increased the number of newly described cyanobacterial taxa, highlighting regions that have fewer studies, such as the North and Northeast regions of the country (Menezes et al. 2015).

Environmental sequencing is an important tool for the study of microbial communities, as most microorganisms cannot be cultivated in vitro (Locey and Lennon 2016). However, combining this tool with classical microbiology techniques, through the use of culture media to enrich target organisms, has become an alternative for the study of bacterial groups that would not be identified only through the sequencing of the environmental 16S rRNA gene. In this study, 17 genera of photosynthetic microorganisms were identified in the environmental taxonomic annotation, while after enrichment with selective medium, it was possible to identify 34 different photosynthetic groups. Photosynthetic microorganisms, when associated with microbial consortia, can in many cases act as main primary producers, helping the growth of other microorganisms and creating a community that would not be cultivated independently (Rappé and Giovannoni 2003; Alvarenga et al. 2017). 
The diversity indices (alpha and beta) were also calculated for the consortium samples to analyze the structure and composition of the microbial community enriched in the culture medium. The alpha diversity, analyzed between the sampling points (Pedra Caída and Farinha River), revealed that the richness of ASVs measured by the Chao1 estimator, the diversity estimated by Shannon, and the number of observed ASVs (Supplementary Figure 2) did not demonstrate an effect on the estimated indexes ( $t$-test, $t=-0.3274, \mathrm{df}=$ 7.2985, $p=0.7732 ;$ t-test $t=-0.23153, \mathrm{df}=6.848, p=0.7526 ; t$-test $, t=-0.29907, \mathrm{df}=7.2977, p=0.7732$, respectively).

The non-metric multidimensional scale (nMDS) based on dissimilarities calculated using the Bray-Curtis metric demonstrated dissimilarity (Supplementary Figure 2) between the microbial communities found in the regions of Rio flour and Pedra Caída (Figure 1B). Based on the multivariate permutation analysis of variance (PERMANOVA), the variation in the structure of the microbial community is, in part, explained by the sample points analyzed (ANOSIM, $R=0.3667, p=0.005$ ). This dissimilarity corroborates the diversity directly analyzed in the samples of origin of the enrichments, since in the environmental microbiome, the composition of the communities also varied according to the origin of the samples, which explains part of the variation in the microbial communities in the enrichments.

\subsubsection{Comparison of environmental and enriched microbiomes}

Sequencing was carried out for both the environmental samples collected in the Chapada das Mesas National Park and the samples cultivated and enriched for photosynthetic microorganisms, in order to analyze the possible subsampling of this group of photosynthetic microorganisms in these environments when analyzed only by independent cultivation techniques. A total of 4,792 AVSs were identified (Figure 3A), with only $2.65 \%$ sharing both in the environmental samples and in the microbial consortia.

In samples obtained from environmental sediment, 2,385 ASVs were found exclusively in this treatment. Only 2,280 ASVs were identified in the cultured samples after enrichment. This indicates that $47.57 \%$ of the microbiota could be cultivated when we used a minimal and selective culture medium for photosynthesizing microorganisms.

The large number of ASVs that were identified exclusively in the cultures (Figure $3 \mathrm{~A}$ ) led us to suppose that the microbes cultivated in our experiment may be representative of the rare portion of the sediment microbiota, that is, likely microorganisms that would not be identified only with sequencing of environmental samples owing to their low relative abundance, and therefore, require a very high sequencing coverage and, in practice, often unfeasible to be represented.

It is also important to note that there was a significant increase $(p<0.05)$ in the abundance and diversity of organisms of the Cyanobacteria phylum in samples that were cultivated in BG-11 culture medium, a minimal medium that allows the growth of photosynthetic microorganisms (Figure 3B). These are also part of this portion, which is not very abundant in the environment and would not be identified only with the sequencing of environmental samples. Therefore, enrichment for specific groups and/or in low abundance can be a good alternative for identifying a better view of the microbiota in a broader way. 
Some phyla such as Myxococcota, Firmicutes, Actinobacteria, and Desulfobacterota were present in the environmental samples and were not identified as phyla with an abundance greater than $1 \%$ in the microbial consortia (Figure 3C). With the enrichment of the Cyanobacteria phylum in the intercropping samples, other phyla such as Bacteroidota, Armatimonadota, and Planctomycetota also showed an increase in their respective abundances compared to environmental samples (Figure 3C), suggesting a greater ecological interrelationship between these phyla.

\subsubsection{Functional inference}

The functional inference analysis performed in FAPROTAX aimed to explore the possible metabolic functions associated with environmental samples and samples grown in consortia.

Regarding the functions (Fig. 4) identified in the environmental samples, there were important ecosystem processes, such as highlighted methanotrophy $(p<0.05)$. Bacteria with this metabolic capacity use methane as a source of carbon and energy, thus mitigating net emissions of methane from natural sources (Crevecoeur et al. 2019), which may be associated with the presence of microorganisms belonging to the genera Methylomonas, Methylocaldum, Crenothrix, and Methylomonas, identified in environmental samples. Methylotrophic processes were also highlighted $(p<0.05)$ in samples collected in the park, associated with methanol oxidation, a process related to the identification of microorganisms from the Methylophilaceae, Methylophilus and Paracoccus groups. Furthermore, a function related to the degradation of hydrocarbons was also identified $(p<0.05)$, a process associated with the genus Halomonas, which is known for its metabolic capacity for the degradation of low- and high-molecular-weight PAHs (Govarthanan et al. 2020), naphthalene (Cheffi et al. 2020) and crude oil (Neifar et al. 2019).

The presence of the genera Prevotella, Faecalibacterium, Parabacteroides Eubacterium Ruminococcus, Bacteroides, and Clostridium in samples from the environmental microbiome highlighted the functions of the human gut and mammalian gut $(p<0.05)$ (Fig. 4). These groups are directly associated with the intestinal microbiota of mammals, which suggests a potential fecal contamination in these water bodies (Kiu et al. 2017; Suzuki et al. 2019; Vadde et al. 2019; Cheffi et al. 2020; Guo et al. 2020; Niestępsk et al. 2020). The presence of these species in the park's samples may indicate that tourism in the area may lead to an anthropization of the place through tourism or agricultural activities, which, in the long term, may modify the local ecosystem.

Comparative functional inference analysis between the consortia where there is enrichment of photosynthetic groups compared to the environmental microbiome revealed a significant increase $(p<0.05)$ in functions related to photosynthetic activity, oxygen phototrophy, and nitrogen fixation. This was already an expected result because cyanobacteria and microalgae are microorganisms responsible for shaping the ecosystems in which they are inserted, as they make carbon sources available through photosynthesis (carbon fixation) (Durall and Lindblad 2015) and are directly involved in the cycle of nitrogen (Paerl 2017), carrying out the process of fixing it. Three genera of the Cyanobacteria phylum (Leptolyngbya, Phormidium, and Synechococcus) were identified only after enrichment in the microbial consortia. These groups can act as important participants in the production of biogenic methane in natural environments, as these genera have already been associated with the production of this hydrocarbon during the oxygen photosynthesis process (Bižić et al. 2020), an increased role in consortia $(p<0.05)$. In addition to oxygenic photosynthesis, the SJA-28 
group, belonging to the phylum Chlorobi, which is known as the green sulfur bacteria (GSB) group (Hiras et al. 2016) was also identified in the consortium samples. This strictly anaerobic, non-mobile, phototrophic microbial group is obligatory phototrophs that oxidize reduced sulfur compounds for $\mathrm{CO}_{2}$ fixation through the reverse tricarboxylic acid (rTCA) cycle, a process known as anoxygenic photosynthesis, and can also carry out $\mathrm{N}_{2}$ fixation (Paun et al. 2019).

Cyanobacteria are a group of microorganisms known for their broad metabolic capacity to produce secondary metabolites, including cyanotoxins (Harada 2004). The enrichment of these microorganisms in the consortia demonstrates the diversity present in the park, and serves as an alert to the risk of possible cyanobacteria and microalgae blooms, as the region is accessed by tourists for leisure in rivers and waterfalls. Alves et al. (2020) investigated the factors that favor blooms of photosynthetic microorganisms in artificial lakes and concluded that seasonality, including rainfall and water temperature, as well as hydrological characteristics such as $\mathrm{pH}$ and nutrient availability, are factors that favor blooms in water bodies.

\section{Conclusions}

To understand if techniques that combine culture-dependent and culture-independent approaches would be an alternative to reveal the diversity of cyanobacteria in water bodies in the Chapada das Mesas National Park, we performed environmental sequencing of the 16S rRNA marker gene and inoculated this same sediment in the medium of selective BG-11 culture to enrich the cultures in cyanobacteria, whose culture was later sequenced as well.

From this combined approach of culture-dependent and culture-independent methodologies, we conclude that it is essential to explore the diversity of groups that are in low abundance or that are disadvantaged by current data analysis methodologies, such as lag in sequence deposition in bank data or in the creation of specific primers. We emphasize that of the 49 groups identified, 32 genera of cyanobacteria were only identified after culture and enrichment, a diversity that would not have been detected if both approaches had not been used. Therefore, there is a need to make use of pre-sequencing techniques, such as enrichment in culture to favor the target groups of the study, using specific culture media, design of primers that increase the level of resolution for representatives of low group abundances in certain ecosystems, and post-sequencing techniques, such as prospecting and mining of genomic sequences and the manual or automatic addition of these sequences to reference databases that will be used for taxonomic composition.

\section{Declarations}

\section{Data availability}

All sequences are deposited in GenBank under accession numbers available in Supplementary table S13.

\section{Funding}

This work was supported by Conselho Nacional de Desenvolvimento Científico e Tecnológico (CNPq) [Grant number 421085/2016-9], Fundação de Amparo à Pesquisa e ao Desenvolvimento Científico e Tecnológico do Maranhão (FAPEMA) [Grant numbers UNIVERSAL-00845/16, ESTAGIO-NACIONAL-05333/18] and 
Coordenação de Aperfeiçoamento de Pessoal de Nível Superior (CAPES) [Grant number CAPES FINANCE CODE 001]. ACAB received fellowships from FAPEMA. LSSF and JCS acknowledge the fellowship support received from CNPq.

\section{Competing interests}

The authors declare that the research was conducted in the absence of any commercial or financial relationships that could be construed as a potential conflict of interest.

\section{Authors contribution}

Ana Carolina de Araújo Butarelli and Lucas Salomão de Sousa Ferreira contributed equally to this work.

Hivana Dall'Agnol, Aline Maria Silva, João Carlos Setúbal, and Leonardo Teixeira Dall'Agnol conceived, obtained funding, and supervised this study.

The cultivation, molecular biology and bioinformatic analysis were performed by Ana Carolina de Araújo Butarelli, Lucas Salomão de Sousa Ferreira, Raquel Riyuzo, and Carlos Morais Piroupo.

Ana Carolina de Araújo Butarelli, Lucas Salomão de Sousa Ferreira, Hivana Dall'Agnol, Aline Maria Silva, João Carlos Setúbal, and Leonardo Teixeira Dall'Agnol wrote and revised the manuscript. All authors read and approved the final manuscript.

\section{Ethics Statement}

Authorization for the collection and transport of samples was approved by the Instituto Chico Mendes de Conservação da Biodiversidade (SISBIO 55217-1) and is registered at the Brazilian National Genetic Heritage and Traditional Knowledge Management System (SISGEN) under the license AACEC6D.

\section{Consent to Publish}

Not applicable.

\section{Acknowledgments}

Leonardo Teixeira Dall'Agnol acknowledge the support received from the Brazilian Microbiome Project (https://www.brmicrobiome.org) and the support of the Marie Curie Alumni Association.

\section{References}

1. Allgaier M, Grossart HP (2006) Diversity and seasonal dynamics of Actinobacteria populations in four lakes in Northeastern Germany. Appl Environ Microbiol 72:3489-3497. https://doi.org/10.1128/AEM.72.5.3489-3497.2006

2. Alvarenga DO, Fiore MF, Varani AM (2017) A metagenomic approach to cyanobacterial genomics. Front Microbiol 8:1-16. https://doi.org/10.3389/fmicb.2017.00809 
3. Alves J, Matthiensen A, Muniz Tagliari MS, Petrucio MM (2020) Climate and hydrological processes explain temporal dissimilarity in the phytoplankton community and favor seasonal dominance of harmful and alien algae in a subtropical reservoir. Panam J Aquat Sci 15:225-243

4. Anderson CR, Peterson ME, Frampton RA et al (2018) Rapid increases in soil pH solubilise organic matter, dramatically increase denitrification potential and strongly stimulate microorganisms from the Firmicutes phylum. https://doi.org/10.7717/peerj.6090. PeerJ 2018:

5. Andrews S (2010) FastQC: a quality control tool for high throughput sequence data. Version 0.11. 2. http://www. bioin forma tics. babra ham. ac. uk/proje cts/fastqc

6. Appolinario LR, Tschoeke DA, Rua CPJ et al (2016) Description of Endozoicomonas arenosclerae sp. nov. using a genomic taxonomy approach. Antonie van Leeuwenhoek. Int J Gen Mol Microbiol 109:431-438. https://doi.org/10.1007/s10482-016-0649-x

7. Arias DM, Uggetti E, García-Galán MJ, García J (2017) Cultivation and selection of cyanobacteria in a closed photobioreactor used for secondary effluent and digestate treatment. Sci Total Environ 587588:157-167. https://doi.org/10.1016/j.scitotenv.2017.02.097

8. Azizan AA, Radzi R, Omar WMW et al (2020) First Records of Morphological Diversity and Ecology of Periphytic Cyanobacteria from Tukun River, Penang Forest Reserve, Malaysia Authors : Asmimie Asmawarnie Azizan, Ranina Radzi, Wan Maznah Wan Omar, Peter Convey and Faradina Merican Mohd Sidik Me. Trop Life Sci Res 31:85-105

9. Bhadury P, Singh T (2020) Analysis of Marine Planktonic Cyanobacterial Assemblages From Mooriganga Estuary, Indian Sundarbans Using Molecular Approaches. Front Mar Sci 7. https://doi.org/10.3389/fmars.2020.00222

10. Bižić M, Klintzsch T, lonescu D et al (2020) Aquatic and terrestrial cyanobacteria produce methane. Sci Adv 6:1-10. https://doi.org/10.1126/sciadv.aax5343

11. Bolyen E, Rideout JR, Dillon MR et al (2019) Reproducible, interactive, scalable and extensible microbiome data science using QIIME 2. Nat Biotechnol 37:852-857. https://doi.org/10.1038/s41587-019-0209-9

12. BRASIL. MINISTRY OF THE ENVIRONMENT (MMA) National Registry of Conservation Units - SNUC (2012) http://www.mma.gov.br/areas-protegidas/cadastro-nacional-de-ucs

13. Brenner K, You L, Arnold FH (2008) Engineering microbial consortia: a new frontier in synthetic biology. Trends Biotechnol 26:483-489. https://doi.org/10.1016/j.tibtech.2008.05.004

14. Callahan BJ, McMurdie PJ, Rosen MJ et al (2016) DADA2: High-resolution sample inference from Illumina amplicon data. Nat Methods 13:581-583. https://doi.org/10.1038/nmeth.3869

15. Cavicchioli R, Ripple WJ, Timmis KN et al (2019) Scientists' warning to humanity: microorganisms and climate change. Nat Rev Microbiol 17:569-586. https://doi.org/10.1038/s41579-019-0222-5

16. Cheffi M, Hentati D, Chebbi A et al (2020) Isolation and characterization of a newly naphthalenedegrading Halomonas pacifica, strain Cnaph3: biodegradation and biosurfactant production studies. https://doi.org/10.1007/s13205-020-2085-x. 3 Biotech 10:

17. Crevecoeur S, Ruiz-González C, Prairie YT, del Giorgio PA (2019) Large-scale biogeography and environmental regulation of methanotrophic bacteria across boreal inland waters. Mol Ecol 28:41814196. https://doi.org/10.1111/mec.15223

Page 14/19 
18. Crump BC, Hobbie JE (2005) Synchrony and seasonality in bacterioplankton communities of two temperate rivers. Limnol Oceanogr 50:1718-1729. https://doi.org/10.4319/lo.2005.50.6.1718

19. Durall C, Lindblad P (2015) Mechanisms of carbon fixation and engineering for increased carbon fixation in cyanobacteria. Algal Res 11:263-270. https://doi.org/10.1016/j.algal.2015.07.002

20. Dvořák P, Poulíčková A, Hašler P et al (2015) Species concepts and speciation factors in cyanobacteria, with connection to the problems of diversity and classification. Biodivers Conserv 24:739-757. https://doi.org/10.1007/s10531-015-0888-6

21. Ferreira LS, de Butarelli S, de Sousa AC et al (2021) High-Quality Draft Genome Sequence of Pantanalinema sp. GBBB05, a Cyanobacterium From Cerrado Biome. Front Ecol Evol 9:1-5. https://doi.org/10.3389/fevo.2021.639852

22. Ferris MJ, Hirsch CF (1991) Method for isolation and purification of cyanobacteria. Appl Environ Microbiol 57:1448-1452. https://doi.org/10.1128/aem.57.5.1448-1452.1991

23. Fournier PE, Lagier JC, Dubourg G, Raoult D (2015) From culturomics to taxonomogenomics: A need to change the taxonomy of prokaryotes in clinical microbiology. Anaerobe 36:73-78. https://doi.org/10.1016/j.anaerobe.2015.10.011

24. Gaysina LA, Saraf A, Singh P (2018) Cyanobacteria in Diverse Habitats. Elsevier Inc

25. Genuário DB, De Souza WR, Monteiro RTR et al (2018) Amazoninema gen. Nov., (Synechococcales, Pseudanabaenaceae) a novel cyanobacteria genus from brazilian amazonian rivers. Int J Syst Evol Microbiol 68:2249-2257. https://doi.org/10.1099/ijsem.0.002821

26. Gibbons SM, Jones E, Bearquiver A et al (2014) Human and environmental impacts on river sediment microbial communities. PLoS ONE 9:1-9. https://doi.org/10.1371/journal.pone.0097435

27. Govarthanan M, Khalifa AY, Kamala-Kannan S et al (2020) Significance of allochthonous brackish water Halomonas sp. on biodegradation of low and high molecular weight polycyclic aromatic hydrocarbons. Chemosphere 243:125389. https://doi.org/10.1016/j.chemosphere.2019.125389

28. Guo P, Zhang K, Ma X, He P (2020) Clostridium species as probiotics: Potentials and challenges. J Anim Sci Biotechnol 11:1-10. https://doi.org/10.1186/s40104-019-0402-1

29. Hamouda RAEF, Sorour NM, Yeheia DS (2016) Biodegradation of crude oil by Anabaena oryzae, Chlorella kessleri and its consortium under mixotrophic conditions. Int Biodeterior Biodegrad 112:128-134. https://doi.org/10.1016/j.ibiod.2016.05.001

30. Harada KI (2004) Production of secondary metabolites by freshwater cyanobacteria. Chem Pharm Bull 52:889-899. https://doi.org/10.1248/cpb.52.889

31. He S, Stevens SLR, Chan L-K et al (2017) Ecophysiology of Freshwater Verrucomicrobia Inferred from. mSphere 2:e00277-e00217

32. Hiras J, Wu YW, Eichorst SA et al (2016) Refining the phylum Chlorobi by resolving the phylogeny and metabolic potential of the representative of a deeply branching, uncultivated lineage. ISME J 10:833-845. https://doi.org/10.1038/ismej.2015.158

33. Hugenholtz P, Skarshewski A, Parks DH (2016) Genome-based microbial taxonomy coming of age. Cold Spring Harb Perspect Biol 8:1-12. https://doi.org/10.1101/cshperspect.a018085 
34. IBAMA (2013) Brazilian Institute of Environment and Renewable Natural Resources. Operational Plan to Prevent and Fight Forest Fires in Chapada das Mesas National Park.

http://www.ibama.gov.br/phocadownload/prevfogo/plano_operativo_parna_da_chapada_das_mesas.pdf

35. Ichor T, Okerentugba PO, Okpokwasili GC (2016) Biodegradation of total petroleum hydrocarbon by a consortium of cyanobacteria isolated from crude oil polluted brackish waters of bodo creeks in Ogoniland, Rivers State. Res J Environ Toxicol 10:16-27. https://doi.org/10.3923/rjet.2016.16.27

36. Jung J, Choi S, Jung $\mathrm{H}$ et al (2013) Primers for amplification of nitrous oxide reductase genes associated with firmicutes and bacteroidetes in organic-compound-rich soils. Microbiol (United Kingdom) 159:307315. https://doi.org/10.1099/mic.0.060194-0

37. Kielak AM, Barreto CC, Kowalchuk GA et al (2016) The ecology of Acidobacteria: Moving beyond genes and genomes. Front Microbiol 7:1-16. https://doi.org/10.3389/fmicb.2016.00744

38. Kiu R, Caim S, Alcon-Giner C et al (2017) Preterm infant-associated clostridium tertium, clostridium cadaveris, and clostridium paraputrificum strains: Genomic and evolutionary insights. Genome Biol Evol 9:2707-2714. https://doi.org/10.1093/gbe/evx210

39. Lesack K, Birol I (2018) Nomenclature Errors in Public 16S rRNA Gene Reference Databases. bioRxiv, 441576

40. Locey KJ, Lennon JT (2016) Scaling laws predict global microbial diversity. Proc Natl Acad Sci U S A 113:5970-5975. https://doi.org/10.1073/pnas.1521291113

41. Louca S, Parfrey LW, Doebeli M (2016) Decoupling function and taxonomy in the global ocean microbiome. Science 353(6305):1272-1277

42. Love MI, Huber W, Anders S (2014) Moderated estimation of fold change and dispersion for RNA-seq data with DESeq2. Genome Biol 15:1-21. https://doi.org/10.1186/s13059-014-0550-8

43. Menezes M, Bicudo CE, Moura CW, Alves AM, Santos AA, Pedrini ADG, Silva WJD (2015) Update of the Brazilian floristic list of Algae and Cyanobacteria. Rodriguésia, 66, 1047-1062

44. McMurdie PJ, Holmes S (2013) Phyloseq: An R Package for Reproducible Interactive Analysis and Graphics of Microbiome Census Data. https://doi.org/10.1371/journal.pone.0061217. PLoS One 8:

45. Neifar M, Chouchane H, Najjari A et al (2019) Genome analysis provides insights into crude oil degradation and biosurfactant production by extremely halotolerant Halomonas desertis G11 isolated from Chott El-Djerid salt-lake in Tunisian desert. Genomics 111:1802-1814. https://doi.org/10.1016/j.ygeno.2018.12.003

46. Niestępski S, Harnisz M, Korzeniewska E, Osińska A (2020) Markers specific to bacteroides fragilis group bacteria as indicators of anthropogenic pollution of surface waters. Int J Environ Res Public Health 17:114. https://doi.org/10.3390/ijerph17197137

47. Oksanen AJ, Blanchet FG, Friendly M et al (2019) Vegan. Encycl Food Agric Ethics 2395-2396. https://doi.org/10.1007/978-94-024-1179-9_301576

48. Padilla-Córdova $C$, Mongili $B$, Contreras $P$ et al (2020) 1 This article is protected by copyright. All rights reserved 15:4-6

49. Paerl $H$ (2017) The cyanobacterial nitrogen fixation paradox in natural waters. F1000Research 6:1-6. https://doi.org/10.12688/f1000research.10603.1 
50. Pascault N, Roux S, Artigas J et al (2014) A high-throughput sequencing ecotoxicology study of freshwater bacterial communities and their responses to tebuconazole. FEMS Microbiol Ecol 90:563574. https://doi.org/10.1111/1574-6941.12416

51. Paun VI, Icaza G, Lavin P et al (2019) Total and potentially active bacterial communities entrapped in a late glacial through holocene ice core from scarisoara ice Cave, Romania. Front Microbiol 10:1-19. https://doi.org/10.3389/fmicb.2019.01193

52. Perera IA, Abinandan S, Subashchandrabose SR et al (2019) Advances in the technologies for studying consortia of bacteria and cyanobacteria/microalgae in wastewaters. Crit Rev Biotechnol 39:709-731. https://doi.org/10.1080/07388551.2019.1597828

53. Pudasaini S, Wilson J, Ji M et al (2017) Microbial diversity of browning Peninsula, Eastern Antarctica revealed using molecular and cultivation methods. Front Microbiol 8. https://doi.org/10.3389/fmicb.2017.00591

54. Quast C, Pruesse E, Yilmaz P et al (2013) The SILVA ribosomal RNA gene database project: Improved data processing and web-based tools. Nucleic Acids Res 41:590-596. https://doi.org/10.1093/nar/gks1219

55. Rappé MS, Giovannoni SJ (2003) The Uncultured Microbial Majority. Annu Rev Microbiol 57:369-394. https://doi.org/10.1146/annurev.micro.57.030502.090759

56. Rego A, Raio F, Martins TP et al (2019) Actinobacteria and cyanobacteria diversity in terrestrial Antarctic microenvironments evaluated by culture-dependent and independent methods. Front Microbiol 10:1-19. https://doi.org/10.3389/fmicb.2019.01018

57. Richardson J, Miller C, Maberly SC et al (2018) Effects of multiple stressors on cyanobacteria abundance vary with lake type. Glob Chang Biol 24:5044-5055. https://doi.org/10.1111/gcb.14396

58. Rigonato J, Alvarenga DO, Andreote FD et al (2012) Cyanobacterial diversity in the phyllosphere of a mangrove forest. FEMS Microbiol Ecol 80:312-322. https://doi.org/10.1111/j.1574-6941.2012.01299.x

59. Rinke C, Schwientek P, Sczyrba A et al (2013) Insights into the phylogeny and coding potential of microbial dark matter. Nature 499:431-437. https://doi.org/10.1038/nature12352

60. AK SDM P (2015) Microbial Ecology in the Era of Next Generation Sequencing. J Next Gener Seq Appl 01. https://doi.org/10.4172/2469-9853.s1-001

61. Samad MS, Lee HJ, Cerbin S et al (2020) Niche Differentiation of Host-Associated Pelagic Microbes and Their Potential Contribution to Biogeochemical Cycling in Artificially Warmed Lakes. Front Microbiol 11:1-14. https://doi.org/10.3389/fmicb.2020.00582

62. Sarhan MS, Hamza MA, Youssef HH et al (2019) Culturomics of the plant prokaryotic microbiome and the dawn of plant-based culture media - A review. J Adv Res 19:15-27. https://doi.org/10.1016/j.jare.2019.04.002

63. Semsarilar M, Guthrie JT, Perrier S et al (2016) of nanoreactors measured by TGA [e.g., T. Science 353:1272-1277

64. Spain AM, Krumholz LR, Elshahed MS (2009) Abundance, composition, diversity and novelty of soil Proteobacteria. ISME J 3:992-1000. https://doi.org/10.1038/ismej.2009.43

65. Stevenson BS, Eichorst SA, Wertz JT et al (2004) New strategies for cultivation and detection of previously uncultured microbes. Appl Environ Microbiol 70:4748-4755. 
https://doi.org/10.1128/AEM.70.8.4748-4755.2004

66. Suzuki A, Ueda K, Segawa T, Suzuki M (2019) Fecal microbiota of captive Antillean manatee Trichechus manatus manatus. FEMS Microbiol Lett 366:1-5. https://doi.org/10.1093/femsle/fnz134

67. Tan BF, Ng C, Nshimyimana JP et al (2015) Next-generation sequencing (NGS) for assessment of microbial water quality: Current progress, challenges, and future opportunities. Front Microbiol 6. https://doi.org/10.3389/fmicb.2015.01027

68. Taranu ZE, Gregory-Eaves I, Leavitt PR et al (2015) Acceleration of cyanobacterial dominance in north temperate-subarctic lakes during the Anthropocene. Ecol Lett 18:375-384.

https://doi.org/10.1111/ele.12420

69. Tromas N, Fortin N, Bedrani L et al (2017) Characterising and predicting cyanobacterial blooms in an 8year amplicon sequencing time course. ISME J 11:1746-1763. https://doi.org/10.1038/ismej.2017.58

70. Vadde KK, Feng Q, Wang J et al (2019) Next-generation sequencing reveals fecal contamination and potentially pathogenic bacteria in a major inflow river of Taihu Lake. Environ Pollut 254:113108. https://doi.org/10.1016/j.envpol.2019.113108

71. Vaz MGMV, Genuário DB, Andreote APD et al (2015) Pantanalinema gen. nov. and Alkalinema gen. nov.: Novel pseudanabaenacean genera (Cyanobacteria) isolated from saline-alkaline lakes. Int J Syst Evol Microbiol 65:298-308. https://doi.org/10.1099/ijs.0.070110-0

72. Wang Y, Qian PY (2009) Conservative fragments in bacterial 16S rRNA genes and primer design for $16 \mathrm{~S}$ ribosomal DNA amplicons in metagenomic studies. PLoS ONE 4. https://doi.org/10.1371/journal.pone.0007401

73. Wickham H (2016) ggplot2-Elegant Graphics for Data Analysis. Springer International Publishing. Cham, Switz

74. Xu Z, Te SH, He Y, Gin KYH (2018) The characteristics and dynamics of cyanobacteria-heterotrophic bacteria between two estuarine reservoirs - Tropical versus subtropical regions. Front Microbiol 9:1-13. https://doi.org/10.3389/fmicb.2018.02531

75. Zarraonaindia I, Owens SM, Weisenhorn P et al (2015) The soil microbiome influences grapevineassociated microbiota. https://doi.org/10.1128/mBio.02527-14. MBio 6:

76. Zehavi T, Probst M, Mizrahi I (2018) Insights into culturomics of the rumen microbiome. Front Microbiol 9:1-10. https://doi.org/10.3389/fmicb.2018.01999

77. Zhubanova AA, Ernazarova AK, Kaiyrmanova GK et al (2013) Construction of cyanobacterial-bacterial consortium on the basis of axenic cyanobacterial cultures and heterotrophic bacteria cultures for bioremediation of oil-contaminated soils and water ponds. Russ J Plant Physiol 60:555-562. https://doi.org/10.1134/S1021443713040183

\section{Figures}

\section{Figure 1}


Structural diversity of the environmental microbiome. (A) Alpha diversity of the environmental microbiome (including the metrics): observed, Chao1 and Shannon, plotted in Box-plot; (B) beta diversity, using the Bray Curtis metric, plotted in NMDS.

\section{Figure 2}

Relative abundance of photosynthetic genera identified in the environmental microbiome and in consortia. The circles represent the abundance of groups in each treatment and the colors differentiate the analyzed treatments.

\section{Figure 3}

Venn diagram with the number of shared and unique ASVs found in each treatment (A) Relative abundance of the Cyanobacteria phylum among microbiomes. (B) Ten most abundant phyla considering the two analysis approaches (>1\%). (C) Comparison between the diversity observed in the environmental and enriched microbiome.

\section{Figure 4}

Functional classes identified through functional inference performed using FAPROTAX software. Comparison between the enriched microbiome (C1-C12), in green and environmental (T1-T12), in blue. Bubbles represent the increase or decrease of the score in relation to treatments. Significantly different functions are shown in supplementary table 12 .

\section{Supplementary Files}

This is a list of supplementary files associated with this preprint. Click to download.

- SupplementaryInformationCMNP.docx 